\title{
MODELOS COMPARTIMENTAIS INVESTIGADOS POR PROCESSOS ESTOCÁSTICOS
}

\author{
Alisson Daniel de Macedo Vitor*, Érica Keith Aparecida Luiz Morais*, Erivelton \\ Geraldo Nepomuceno ${ }^{\dagger}$ \\ ${ }^{*}$ Universidade Federal de São João del-Rei \\ Programa de Pós-Graduação em Engenharia Elétrica - Parceria Ampla CEFET-UFSJ \\ São João del-Rei, MG, Brasil \\ ${ }^{\dagger}$ Dept. Electrical and Eletronic Engineering, Imperial Colege London \\ London SWr 2BT, UK \\ Emails: amv.projetos@gmail.com, ericamoraisarruda@gmail.com, \\ e.nepomuceno@imperial.ac.uk
}

\begin{abstract}
Compartmental models have been targets of scientific research in various areas of knowledge, eg, Engineering, Physics, Mathematics, Ecology and Medicine. In this work, we investigated the dynamics of a compartmental epidemiological model through stochastic differential equations. The model was built from the classical model compartmental Susceptible-Infected-Recovered and the results were obtained by Monte Carlo simulation. The mean values of the population of susceptible and infected are showed and investigated the dynamics of classical model and stochastic model by varying the rate of strength of an infection.
\end{abstract}

Keywords - Stochastic Dynamics, Stochastic Differential Equations, Compartmental Models, Monte Carlo

Resumo - Modelos compartimentais têm sido alvos da investigação científica em diversas áreas do conhecimento como, por exemplo, Engenharias, Física, Matemática, Ecologia e Medicina. Neste trabalho, é investigada a dinâmica de um modelo compartimental epidemiológico através de equações diferenciais estocásticas. O modelo foi construído a partir do modelo clássico compartimental Suscetível-Infectado-Recuperado e os resultados foram obtidos por simulação de Monte Carlo. São apresentados os valores médios da população de suscetíveis e infectados e investigada a dinâmica dos modelos clássico e estocástico através da variação da taxa que mede a força de uma infecção.

Palavras-chave- Dinâmica Estocástica, Equações Diferencias Estocásticas, Modelos Compartimentais, Simulação de Monte Carlo.

\section{Introdução}

A classificação dos indivíduos em estados ou compartimentos é a abordagem mais utilizada para estudar sistemas epidemiológicos. Kermack e McKendrick (Kermack and McKendrick, 1927) propuseram um modelo, baseado em equações diferencias ordinárias não-lineares, para investigar a propagação de uma doença infecciosa que se espalha rapidamente por um população. Usualmente denomina-se o modelo de Kermack e MacKendrick de modelo SIR. Também utilizaremos o termo modelo SIR clássico ou modelo SIR original para diferenciá-lo do modelo SIR estocástico proposto por este trabalho.

O modelo SIR gerou contribuições importantes na epidemiologia matemática, dentre as quais destacam-se a ação de massa e existência de um limiar para a erradicação da doença e continua sendo estudado até os dias de hoje (Morais, 2012), (Satsuma et al., 2004). Há, porém, contribuições que ainda podem ser feitas e que não são suficientemente explicadas pelo Modelo SIR Clássico, como, por exemplo, a persistência de doenças infecciosas e a influência da distribuição espacial e temporal dos agentes indivíduos na propagação da doença.

Para entender e prever o comportamento de uma epidemia é necessário a elaboração de um modelo matemático capaz de tais previsões. A dinâmica do modelo SIR é bem representada se se considera a distribuição homogênea das indivíduos . Entretanto, quando se considera iterações estocásticas existentes entre os indivíduos, que são típicas de sistemas multiagentes, percebe-se que é necessário modificar o modelo SIR clássico para chegar em resultados de interesse.

Diferentes modificações têm sido aplicadas no modelo SIR original a fim de acrescentar variáveis estocásticas em sua estrutura: (Zhang et al., 2013) incorpora uma distribuição de escala livre e rede estruturada num modelo estocástico SIR analisado através de redes complexas; (Ji et al., 2012) investiga um modelo SIR, que sofre uma perturbação estocástica, através de funcionais estocásticos de Lyapunov; (Schuetz et al., 2008) cria um modelo SIR estocástico baseados na teoria de grafos, representado os indivíduos e suas interações por nós e conexões. Os autores mapeiam o conjunto de equações mestras do modelo SIR estocástico numa formulação quântica em termos de operadores de spin de tal forma que é possível obter uma solução exata para o sistema para condições iniciais arbitrárias. Os resultados da solução exata são comparados com simulações numéricas; (Tornatore et al., 2005) propõe um modelo esto- 
cástico SIR com e sem intervalo de tempo de distribuição e investiga sua estabilidade. As simulações numéricas mostram que a introdução de ruído modificam os limites para uma epidemia ocorrer e identificar esses limites no trabalho.

Neste trabalho, é investigada a dinâmica de um modelo compartimental epidemiológico através de equações diferenciais estocásticas. O modelo obtido foi construído a partir Modelo Clássico SIR através da inclusão de um termo estocástico nas equações diferencias que descrevem os indivíduos suscetíveis e infectados. A dinâmica é investigada através da análise do termo dos conjunto de equações que representa a força da infecção. Ambos os modelos foram analisados e os resultados são apresentados, assim como comparações entre eles. para o modelo estocástico, em particular, as equações diferenciais foram simuladas por processo de Monte Carlo e foram apresentos como resultados termos médios da simulação.

\section{Modelo SIR Original}

No modelo SIR divide-se a população em três classes: a classe dos suscetíveis, S, composta por indivíduos que podem contrair a doença através de contato com indivíduos infectados; a classe do infectados , I, formada por indivíduos que têm a doença e podem transmití-la; e a classe dos removidos ou recuperados, $R$, que passaram pela doença e não são mais nem suscetíveis nem infectados, de modo que eles ou morreram ou se recuperaram. Desta forma, assume-se a cura confere imunidade (Monteiro, 2010). Sejam S(t), I(t), R(t) os números ou indivíduos de cada classe no instante $t$. O modelo SIR pode ser expresso como (Nepomuceno, 2005) :

$$
\begin{gathered}
\frac{d S}{d t}=\mu N-\mu S-\beta I S / N, \\
\frac{d I}{d t}=\beta I S / N-\gamma I-\mu I, \\
\frac{d R}{d t}=\gamma I-\mu R+p \mu N,
\end{gathered}
$$

em que o estado inicial é $(S(0), I(0), R(0)) \geq 0$, $S, I, R$ são o número de indivíduos suscetíveis, infectados e recuperados, respectivamente; $N$ é o número total de indivíduos, $\beta$ é a taxa de transmissão; $\mu$ é taxa de novos suscetíveis, sendo que para manter a população contante faz-se a taxa de mortalidade $d=\mu ; \gamma$ é a taxa de indivíduos infectados que são recuperados e $S(t)+I(t)+R(t)=N$. Tais constantes positivas caracterizam a interação entre o indivíduo infeccioso e a população. Neste modelo assume-se que:

- o número de infectados aumenta segundo uma taxa proporcional ao produto entre o número de infectados e o de suscetíveis, sendo os suscetíveis perdidos nesta mesma taxa;
- a taxa de passagem dos infectados para a classe dos recuperados é proporcional ao número de infectados;

- o período de incubação é desprezível, de maneira que um suscetível que contrai a doença torna-se imediatamente infectado;

- as três classes estão uniformemente distribuídas no espaço. Assim, as taxas de encontro independem da localização geográfica, o que permite modelar este sistema por equações diferenciais ordinárias.

Analisam-se as soluções do sistema 1 por meio do conceito de fator de reprodutividade basal, definido a partir dos parâmetros $\beta$, $\gamma$ e o estado inicial $(S(0), I(0), R(0))$. Da segunda equação de Eq. 1 temos:

$$
\left.\frac{d I(t)}{d t}\right|_{t=0}=I(0)(\beta S(0)-\gamma-\mu) .
$$

Desta forma, a doença de espalha e o número de suscetíveis diminui consideravelmente se $d I(t) / d t>0$, ou seja, se $\beta S(0)>\gamma-\mu$. Se $\beta S(0)<\gamma+\mu$ não há infecção. Portanto, o fator de reprodutividade basal é definido como:

$$
R_{0} \equiv \frac{\beta S(0)}{\gamma+\mu}
$$

e verifica-se que há epidemia se $R_{0}>1$ ou não há epidemia se $R_{0}<1$.

Dividindo as equações em 1 por $N$ e desconsiderando $R$, que pode ser obtido por $R=N-S-I$ têm-se:

$$
\begin{gathered}
d s / d t=\mu-\mu S-\beta i s, \quad S(0)=s_{0} \geq 0, \\
d i / d t=\beta i s-\gamma i-\mu i, \quad i(0)=I_{0} \geq 0,
\end{gathered}
$$

assumindo que $\beta I / N=\beta i$ como sendo a média do número de contatos com infectados por unidade de tempo de um indivíduo infectado.

\section{Modelo SIR Estocástico}

Um processo estocástico no espaço de probabilidade $(\Omega, F, P)$ é uma coleção indexada de $X_{t}, t \in I$ de variáveis aleatórias. Para investigação de equações diferenciais estocásticas considera-se $t$ como tempo e o conjunto de índices é usualmente um intervalo de tempo da forma $[0,+\infty),[-\infty,+\infty]$ ou $[a, b]$ (processos estocásticos em tempo contínuo). Noutras situações, $I$ pode ser o conjunto dos inteiros ou inteiros não-negativos (processos estocásticos em tempo discreto). Como cada variável aleatória $X_{t}=X_{t}(\omega)$ é uma função do "acaso" $\omega \in \Omega$, um processo estocástico pode ser considerado uma função de duas variáveis: $t \in I$ e $\omega \in \Omega$, ou seja, é uma função do tempo e do "acaso".

Se fixarmos o "acaso" $\omega$ obtemos uma função 
apenas do tempo denominada trajetória do processo estocástico. Um processo estocástico pode, portanto, ser interpretado como uma coleção de trajetórias, uma para cada estado do "acaso" $\omega$.

Uma equação diferencial estocástica pode ser escrita como (Oksendal, 2007):

$$
\frac{d X}{d t}=b\left(t, X_{t}\right)+\sigma\left(t, X_{t}\right) \cdot W_{t},
$$

em que $b$ e $\sigma$ são funções dadas e $W_{t}$ é o termo estocástico que representa um ruído branco. Assumise que $W_{t}$ possui as seguintes propriedades:

- $t_{1} \neq t_{2} \Rightarrow W_{t_{1}}$ e $W_{t_{2}}$ são independentes;

- $\left\{W_{t}\right\}$ é estacionário, ou seja, a distribuição conjunta de $\left\{W_{t_{1}+t}, \ldots, W_{t_{k}+t}\right\}$ não depende de $t$

- $E\left[W_{t}\right]=0$ para todo $t$

As propriedades citadas de $W_{t}$ descrevem algumas características do processo de Wiener ou movimento browniano. Maiores detalhes do processo de Wiener podem ser obtidos em (Morais, 2012), (Oksendal, 2007) e (Braumann, 2005).

O modelo SIR pode ser modificado de modo que haja a inserção de variáveis estocásticas na sua descrição. Sejam as matrizes esperança matemática, $E$, e covariância, $C O V$, respectivamente dadas por:

$$
E=\sum_{i=1}^{N} p_{i} \lambda_{i}
$$

e

$$
C O V=\sum_{i=1}^{N} p_{i} \lambda_{i} \lambda_{i}^{T},
$$

em que $\lambda_{i}$ é a mudança de estado e $p_{i}$ é a probabilidade desta mudança. Aplicando 6 e 7 no sistema de equações 4 , obtêm-se:

$$
\begin{gathered}
E=\left[\begin{array}{c}
\mu-\mu S-\beta i \\
\beta i s-\gamma i-\mu i
\end{array}\right] \\
C O V=\left[\begin{array}{cc}
\mu-\mu s-\beta i & -\beta s i \\
-\beta s i & \beta i s+\gamma i+\mu i
\end{array}\right] .
\end{gathered}
$$

E o sistema de equações 4 pode ser reescrito com a adição do termo estocástico:

$$
\begin{aligned}
d s / d t & =\mu-\mu s-\beta i s+B_{11} \frac{d W_{1}}{d t}, S(0)=s_{0} \geq 0, \\
d i / d t & =\beta i s-\gamma i-\mu i+B_{22} \frac{d W_{2}}{d t}, i(0)=I_{0} \geq 0,
\end{aligned}
$$

em que $W_{1}$ e $W_{2}$ são processos estocásticos de Wiener e o vetor $\mathrm{B}$ é a raiz quadrada da matriz de covariância $C O V$.

\section{Resultados}

Verificou-se o comportamento dinâmico do modelo SIR clássico a partir da variação do valor da taxa de infecção $\beta$. Foram considerados os seguintes parâmetros: $\beta=0,002 ; 0,4 ; 0,5 ; 0,7, \gamma=0,4$, $\mu=0,016$. O número de indivíduos foi normalizado, ou seja, $N=1$. As condições iniciais são $85 \%$ da população no estado de suscetíveis e 5\% no estado de infectados.
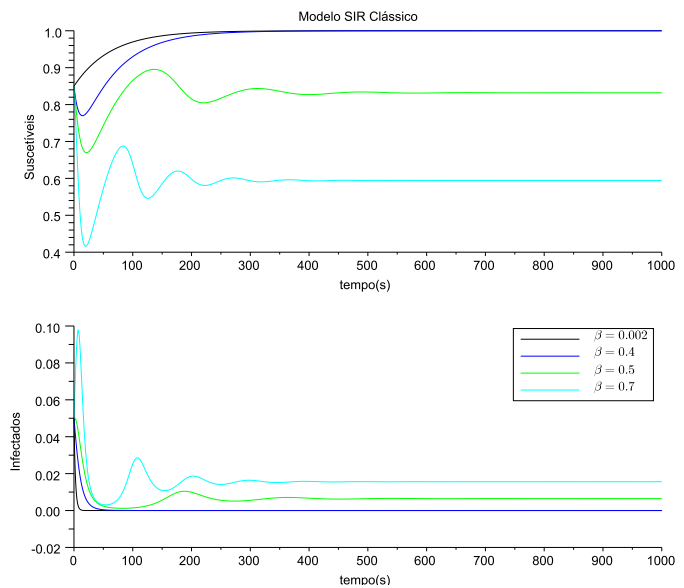

Figura 1: Modelo SIR Clássico. Com a taxa de infecção $\beta=0,002$ e $\beta=0,4$ da população não é possível estabelecer uma epidemia, ou seja, a força da infecção é pequena para estabelecer o contágio. Para $\beta=0,4$ a curva de suscetíveis diminui um pouco, mas logo retorna para o valor máximo da população. Já para as taxa de infecção $\beta=0,5$ e $\beta=0,7$ a doença de espalha e o número de suscetíveis cai rapidamente. Após a infecção estabelecer-se, a partir de $200 s$, a fase endêmica começa a aparecer, a partir da qual não se observa mais oscilações na curva. Parâmetros: $\beta=0,002 ; 0,4 ; 0,5 ; 0,7, \gamma=0,4, \mu=0.016$, $N=1$. Rotina: SIRCLASSICO.SCE

Para $\beta=0,002$ e $\beta=0,4$ obtêm-se $R_{0}=0,004$ e $R_{0}=0,850$, respectivamente, e a infecção não é capaz de atingir uma grande parte da população. Já para $\beta=0,5$ e $\beta=0,7$ têm-se $R_{0}=1,062$ e $R_{0}=1,487$ e o consequente estabelecimento da epidemia. Neste caso, a Fig. 1 mostra uma solução do modelo SIR com faixa de tempo suficiente para apresentar as fases epidêmicas e endêmicas. O rápido decrescimento da população de suscetíveis corresponde à fase epidêmica; o nível de infecção cresce rapidamente, levando o número de suscetíveis a um nível inferior ao equilíbrio em estado permanente. ) A partir de $200 s$ dá-se lugar à fase endêmica onde se observa um padrão senoidal ligeiramente amortecido em torno do ponto de equilíbrio.

Para simulação do modelo SIR estocástico foram utilizados os mesmos parâmetros do modelo 
SIR clássico. O processo foi inicializado com $85 \%$ da população no estado de suscetíveis e $5 \%$ no estado de infectados. Foi utilizado o método de Monte Carlo para obter o valor de cada indivíduo suscetível e infectado: para cada instante $t$ foi simulada 1000 iterações para cada equação do sistema 10 e calculada a média dessas iterações para obter um indivíduo suscetível e um indivíduo infectado.

O sistema de equações 10 gera um conjunto de trajetórias do modelo SIR estocástico proposto. As trajetórias mudam em cada simulação, como é de se esperar de um processo dessa natureza. A Fig. 2 mostra uma trajetória para $\beta=0,002$ de valores médios obtidos para a população de suscetíveis e infectados.
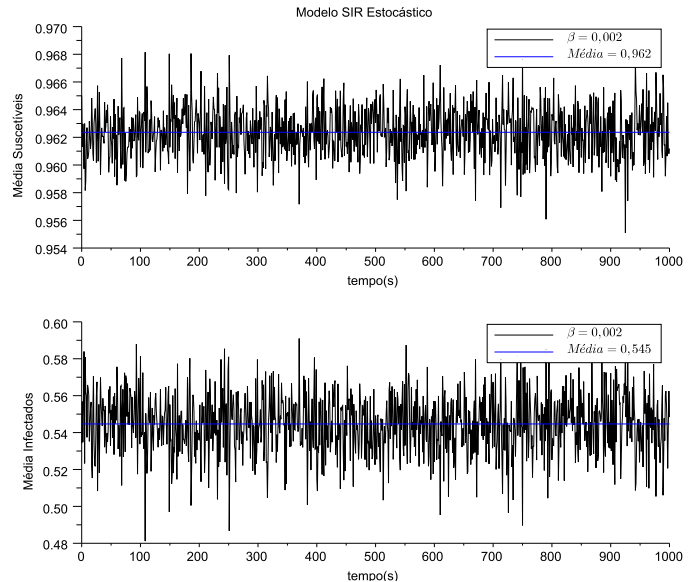

Figura 2: Modelo SIR Estocástico. Uma trajetória para $\beta=0,002$. São aprasentados os valores médios de 1000 iterações para cada indivíduo suscetível e infectado. Com a taxa de infecção $\beta=0,002$ da população não é possível estabelecer uma epidemia, mas diferentemente do modelo clássico, o número de suscetíveis não tende ao valor total da população e o número de infectados não tende a zero. Observa-se uma variação dos valores médios ao torno de 0,966 e 0,517 para os indivíduos suscetíveis e infectados, respectivamente. Parâmetros: $\beta=0,002, \gamma=0,4, \mu=0.016$, $N=1$. Rotina: SIRESTOCASTICO.SCE

A Fig. 3 mostra os resultados das simulações para $\beta=0,25$, com os valores médios de suscetíveis e infectados em torno de 0,437 e 0,587 , respectivamente, e A Fig. 4 mostra os resultados das simulações para $\beta=0,40$, com os valores médios de suscetíveis e infectados em torno de $0,441 \mathrm{e}$ 0,582 .

O comportamente médio do modelo estocástico comporta-se qualitativamente semelhante ao modelo clássico, com a variação da população de suscetíveis e infectados de acordo com o valor da taxa de infecção $\beta$. Em ambos os modelos, à medida que cresce o valor da taxa de infecção, a população de suscetíveis diminiu e a população de
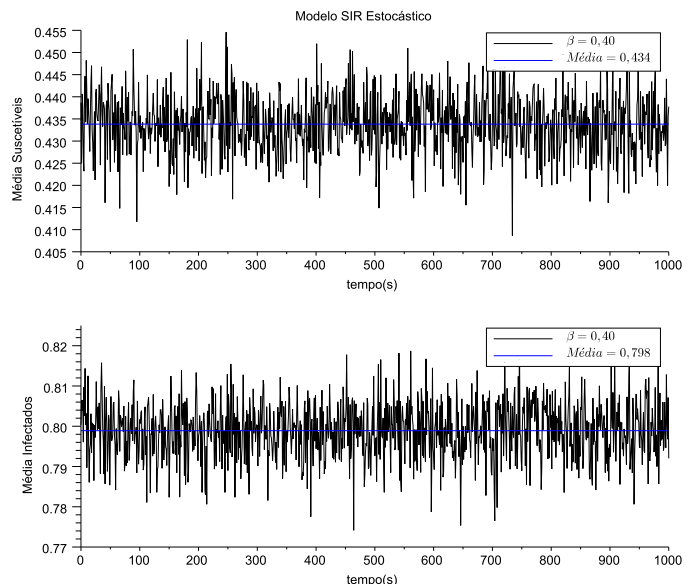

Figura 3: Modelo SIR Estocástico. Uma trajetória para $\beta=0,40$. São aprasentados os valores médios de 1000 iterações para cada indivíduo suscetível e infectado, respectivamente. Com a taxa de infecção $\beta=0,40$ da população tem-se $R_{0}=0,850$. A variação dos valores médios acorre em torno de 0,437e 0,587 para os indivíduos suscetíveis e infectados, respectivamente. Parâmetros: $\beta=0,40, \gamma=0,4, \mu=0.016, N=1$. Rotina: SIRESTOCASTICO.SCE
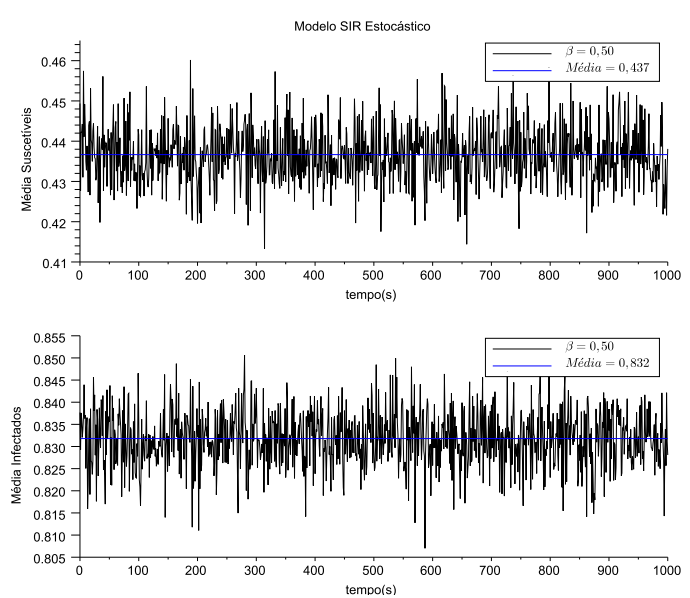

Figura 4: Modelo SIR Estocástico. Uma trajetória para $\beta=0,50$. São aprasentados os valores médios de 1000 iterações para cada indivíduo suscetível e infectado, respectivamente. Com a taxa de infecção $\beta=0,40$ da população tem-se $R_{0}=1,062$. A variação dos valores médios acorre em torno de 0,441 e 0,582 para os indivíduos suscetíveis e infectados, respectivamente. Parâmetros: $\beta=0,40, \gamma=0,4, \mu=0.016, N=1$. Rotina: SIRESTOCASTICO.SCE 
infectados aumenta. Há, entretanto, algumas divergências. Por exemplo, no modelo clássico, para $\beta=0,70$ a população de suscetíveis atinge um primeiro mínimo em $25 \mathrm{~s}$ de $42 \%$, um segundo mínimo em $120 s$ de $56 \%$ enquanto a população de infectados atinge os valores máximos $10 \%$ e $3 \%$ nos mesmos instantes de tempo. Após 200s, o modelo clássico apresenta a fase endêmica da doença com $58 \%$ e $2 \%$ da população de suscetíveis e infectados, respectivamente, mostrando que a infecção não se extingue completamente. No modelo estocástico, para $\beta=0,40$ tem-se uma população média de suscetíveis de $43,4 \%$ e uma população média de infectados de $79,8 \%$. Ou seja, o modelo estocástico não prevê a extinção da infecção mesmo com $R_{0}<1$, pois existe uma probabilidade não-nula de que a infecção se espalhe e contamine uma parcela grande da população. Também não é possível falar de comportamento de fase endêmica no modelo estocástico, pois ele não apresenta amortecimento nas oscilações. Verifica-se que o modelo estocástico é sensível ao valor da taxa de infecção $\beta$; para $\beta>0,50$ não se percebe variações significativas na população média de suscetíveis. Tais observações constam na pauta de investigação da pesquisa que gerou este trabalho cujos resultados são preliminares. Pretende-se ainda aprofundar a pesquisa sobre a dinâmica estocástica desse modelo.

\section{Conclusão}

O modeo SIR clássico é um exemplo de modelos compartimentais e sua investigação tem sido utilizada para entender como populações de indivíduos com características diferentes interagem entre si. Aos modelos compartimentais pode-se incluir elementos estocásticos para aproximá-los de situações mais reais e propor então técnicas de controle ou análise de estabilidade. Diferentes abordagens de processos estocásticos em modelos compartimentais podem ser realizadas: investigação através de redes complexas aleatórias, modelagem por processos de Markov, modelagem por métodos de perturbação, dentre outros. Neste trabalho, procurou-se investigar a dinâmica do modelo SIR incluido ao modelo original termos estocásticos descritos por equações diferenciais estocásticas. Observou-se que o modelo estocástico proposto comporta-se qualitativamente semelhante ao modelo clássico SIR, mas ainda apresenta limitações para investigação de valores maiores da taxa de infecção da população. O trabalho remete para futuras investigações sobre dinâmica estocástica em modelos compartimentais.

\section{Agradecimentos}

Os autores agradecem à FAUF-UFSJ, CNPq e Capes pelo incentivo financeiro no desenvolvimentos das pesquisas. Agradecem ainda ao Programa de
Pós-Graduação em Engenharia Elétrica, parceira ampla entre o Centro Federal de Ensino Técnico de Minas Gerais e a Universidade Federal de São João del Rei.

\section{Referências}

Braumann, C. A. (2005). Uma introdução às equações diferenciais estocásticas e aplicações, Sociedade Portuguesa de Estatística, Ericeira, Portugal, p. 190.

Ji, C., Jiang, D. and Shi, N. (2012). The behavior of an sir epidemic model with stochastic perturbation, Stochastic Analysis and Applications $\quad 30(5)$ : $\quad 755-773 . \quad$ DOI: 10.1080/07362994.2012.684319

Kermack, W. and McKendrick, A. (1927). A contribution to the mathematical theory of epidemics, Proceedings of the Royal Society of London Series A Mathematical and Physical Sciences A115: 700-721. DOI: 10.1098/rspa.1927.0118

Monteiro, L. H. A. (2010). Sistemas Dinâmicos Complexos, São Paulo: Editora Livraria da Física.

Morais, E. K. A. L. (2012). Representação matemática do modelo baseado em indivíduos: uma aplicação em sistemas epidemiologicos., Master's thesis, Programa de Pós-Graduação em Engenharia Elétrica, associação ampla entre a Universidade Federal de São João DelRei e o Centro Federal de Educação Tecnológica de Minas Gerais, São João del Rei, Minas Gerais, Brasil.

Nepomuceno, E. G. (2005). Dinâmica, Modelagem e Controle de Epidemias, $\mathrm{PhD}$ thesis, Programa de Pós-Graduação em Engenharia Elétrica, Universidade Federal de Minas Gerais, Belo Horizonte, Brasil.

Oksendal, B. (2007). Stochastic Differential Equations - An Introduction with Applications, 6 th edn, Springer.

Satsuma, J., Willox, R., Ramani, A. and Grammaticos, B. Carstea, A. S. (2004). Extending the SIR epidemic model, Physica A-Statistical Mechanics and its Applications 336(3-4): 369-375.

Schuetz, G. M., Brandaut, M. and Trimper, S. (2008). Exact solution of a stochastic susceptible-infectious-recovered model, Physical Review E 78(6): 061132. DOI: 10.1103/PhysRevE.78.061132

Tornatore, E., Buccellato, S. M. and Vetro, P. (2005). Stability of a stochastic $\{$ SIR $\}$ system, Physica A: Statistical Mechanics and its Applications 354(0): 111 - 126.

URL: 1 
Zhang, H., Guan, Z.-H., Li, T., Zhang, X.-H. and Zhang, D.-X. (2013). A stochastic sir epidemic on scale-free network with community structure, Physica A-statistical Mechanics and Its Applications 392(4): 974-981. 